\title{
Perceived Adequacy of Tangible Social Support and Associations with Health Outcomes Among Older Primary Care Patients
}

\author{
Rachel $\mathrm{O}^{\prime}$ Conor, $\mathrm{PhD}, \mathrm{MPH}^{7}$, Julia Yoshino Benavente, $\mathrm{MPH}^{7}$, Marina Arvanitis, $\mathrm{MD}^{7}$, \\ Laura M. Curtis, $M S^{l}$, Kamal Eldeirawi, PhD, $R N^{2}$, Romana Hasnain-Wynia, $P h D^{3}$, \\ Alex D. Federman, MD, MPH$H^{4}$, Jeni Hebert-Beirne, $\mathrm{PhD}, \mathrm{MPH}^{5}$, and Michael S. Wolf, \\ $\mathrm{PhD}, \mathrm{MPH}^{\top}$
}

\begin{abstract}
'Division of General Internal Medicine and Geriatrics, Feinberg School of Medicine, Northwestern University, Chicago, IL, USA; ${ }^{2}$ College of Nursing, University of Illinois at Chicago, Chicago, IL, USA; ${ }^{3}$ Office of Research, Denver Health and Hospital Authority, Denver, CO, USA; ${ }^{4}$ Division of General Internal Medicine, Icahn School of Medicine at Mount Sinai, New York, NY, USA; ${ }^{5}$ Division of Community Health Sciences, School of Public Health, University of Illinois at Chicago, Chicago, IL, USA.
\end{abstract}

\begin{abstract}
BACKGROUND: The availability and adequacy of tangible social support may be critical to older adults managing multiple chronic conditions, yet few studies have evaluated the perceived adequacy of needed tangible support and its relation to health outcomes.
\end{abstract}

OBJECTIVE: We investigated the association between unmet, tangible social support needs, health status, and urgent healthcare use among community-dwelling older adults.

DESIGN: Cross-sectional analysis.

PARTICIPANTS: English-speaking older adults $(n=469)$ who participated in the Health Literacy and Cognitive Function cohort study.

MAIN MEASURES: Perceived adequacy of tangible social support was measured using a brief, validated scale that determined (1) if an individual needed assistance managing his or her health, and (2) if yes, whether this need was met. Health status was measured using physical function, depression, and anxiety PROMIS short-form instruments. Urgent healthcare utilization (emergency department and hospitalization) was self-reported for the past 12 months.

KEY RESULTS: Participants' mean age was 69 years; 73\% were women and 31\% were African American, and $16 \%$ identified unmet support needs. Unmet support needs were associated with worse physical $(\beta-6.32$; 95\% CI $-8.31,-4.34$ ) and mental health (anxiety: $\beta$ 3.84; 95\% CI 1.51, 6.17; depression: $\beta$ 2.45; 95\% CI $0.32,4.59)$ and greater urgent healthcare utilization (ED: OR 2.86; 95\% CI 1.51, 5.41; hospitalization: OR 3.75; 95\% CI 1.88, 7.50).

CONCLUSIONS: Perceived unmet support needs were associated with worse health status and greater urgent healthcare use. Primary care practices might consider screening older patients for unmet tangible support

$\overline{\text { Prior Presentations These data were presented at the } 2018 \text { Society of }}$ General Internal Medicine meeting, April 12, 2018, Denver, CO.

Received July 5, 2018

Revised December 12, 2018

Accepted April 5, 2019

Published online June 26, 2019 needs, although appropriate responses should first be established if unmet needs are identified.

KEY WORDS: social support; aging; patient-reported outcomes.

J Gen Intern Med 34(11):2368-73

DOI: $10.1007 / \mathrm{s} 11606-019-05110-7$

๑) Society of General Internal Medicine 2019

\section{INTRODUCTION}

Estimates suggest that between 62 and $80 \%$ of older adults are managing multiple $(\geq 2)$ chronic conditions, ${ }^{1,2}$ which often requires a range of self-management responsibilities in order to maintain one's health. This includes, but is not limited to, adhering to prescribed drug regimens, maintaining recommended diets, engaging in regular physical activity, selfmonitoring symptoms, and keeping routine appointments with healthcare providers. These tasks require significant knowledge and skills, as well as an ability to organize and plan, critically think, and effectively communicate with healthcare providers. The long-term persistence of such self-care demands poses significant challenges for patients over time. Thus, with increasing care needs and complex self-care regimens, older adults may benefit from additional assistance.

Social support is commonly cited as an important determinant of self-management behaviors ${ }^{3,4}$ and health outcomes. ${ }^{5}$ However, social support is multidimensional and influences health outcomes via multiple pathways, ${ }^{6}$ and due to variation in measurement, the evidence is fragmented, making it difficult to discern what dimension of social support is best for chronically ill older adults. ${ }^{7,8}$ Within the context of chronic disease self-management, "tangible support," the provision of direct material aid or other concrete assistance may be more predictive of better health outcomes than other forms of support, as a seminal meta-analysis found tangible support to demonstrate a greater effect on treatment adherence than other forms of support including emotional support or social 
network size. ${ }^{4}$ Yet further complicating the utility of available tangible support is the complexity of chronic disease selfmanagement, as those providing assistance must also have a range of skills and competencies to accurately assist with older adults' self-care regimens.

Surprisingly, few empirical investigations have considered the perceived adequacy of tangible support and its relationship to health outcomes and urgent healthcare utilization. The studies identified as measuring the subjective perceptions of social support in a recent systematic review of social relationships and health services use among older adults captured perceptions of availability of support and loneliness, rather than the perceptions about the adequacy of the support received. ${ }^{7}$ Outside of this review, one investigation has assessed the perceived adequacy of tangible support and health outcomes; patients with coronary artery disease who reported a perceived lack of needed assistance had greater rates of mortality and decline in physical functioning than individuals whose perceived needs for assistance were met. ${ }^{9}$ Unmet needs for social support were also associated with less engagement in diabetes self-care activities among Korean adults with diabetes. ${ }^{10}$ These investigations provide evidence of the importance of considering the adequacy of needed support, but has not been extended to a general population. In order to fill this gap, we sought to evaluate the association between unmet social support needs and older adults' health status and urgent healthcare utilization.

\section{METHODS}

We conducted a cross-sectional secondary data analysis using data collected from the Health Literacy and Cognitive Function among Older Adults study (R01AG030611, referred to as "LitCog"). The primary variables of interest were perceived adequacy of tangible support, health status, and urgent healthcare utilization. The study was approved by the Institutional Review Board at Northwestern University Feinberg School of Medicine.

\section{Sample and Procedure}

LitCog is a prospective cohort study investigating changes in health literacy over time and its relation to cognitive function and performance on healthcare tasks. ${ }^{11}$ The sample was recruited from one academic general internal medicine practice and six federally qualified health centers in Chicago, IL, between August 2008 and August 2015. A description of the systematic recruitment procedures has been previously published. ${ }^{11,12}$ Patients were eligible if they (1) were between the ages of 55 and 74, (2) spoke English, (3) had adequate cognitive capacity, as defined by $\leq 2$ errors on the 6 -item screener, ${ }^{13}$ and (4) sought regular care (defined as 2 clinic visits within the past 2 years) from study sites. The sample was purposefully recruited at a younger age to capture and follow the onset of cognitive decline. Research coordinators administered structured in-person interviews with enrolled participants in a private room. Participants completed follow-up interviews every 3 years and data for these analyses were collected at the third follow-up interview, which took place 6 years following their baseline interview.

\section{Measures}

Adequacy of Tangible Social Support. Adequacy of tangible social support was measured using a brief, validated scale to identify whether an individual needed assistance managing his or her health, and if this need for assistance was met. ${ }^{9}$ Participants were asked two questions: the first question asked "In the last 6 months, have you needed any kind of extra help at home because your health kept you from taking care of yourself or doing what you usually do?" If respondents answered yes, they were asked a follow-up question "Of the help you got at home, would you say you got: all you needed, most you needed, some you needed, only a little, or none of the help you needed?" To create an initial overall score, participants were classified as (1) no perceived need for help; (2) received sufficient help (need help, but all needs met); (3) more help needed (most needs for help met); and (4) much more help needed (only some, little, or none of needs for help met). Based on the distribution of responses, and following previous literature, individuals were further classified as having unmet needs (more help needed, much more help needed) or adequate support (no perceived help needed, received sufficient help). ${ }^{9}$

Functional Health Status. Functional health status was assessed in terms of both physical and mental health. ${ }^{14}$ Functional health status was assessed using Patient-Reported Outcomes Measurement Information Service (PROMIS) short-form instruments of physical function, depression, and anxiety. ${ }^{14}$ Physical function was measured using the 10-item short-form physical function scale, and depressive and anxiety symptoms were measured with the coinciding 8-item shortform PROMIS scales. A raw score is calculated for each scale with high scores indicating greater ability or more symptoms. Raw scores are translated into a corresponding $t$-score, which rescales the raw score into a standardized score with a mean of 50 and standard deviation of 10 . Therefore, a person with a $t$ score of 40 is one standard deviation below the mean score of the general population of the USA. ${ }^{14}$ PROMIS has established minimally important differences (MID) to provide clinical context for some of the scales. A MID is defined as a difference in score that are large enough to have implications for a patient's treatment or care. ${ }^{15}$ The MIDs range from 4 to 6 points and 3-4.5 points for the physical functioning and anxiety and depressive scales, respectively.

Urgent Healthcare Utilization. Urgent healthcare utilization was assessed by self-report of emergency department (ED) visits and unplanned inpatient hospitalization. During the interviews, patients were asked to self-report if they (1) visited the emergency department in the past 12 months and (2) had an unplanned hospitalization in the past 12 months. Questions 
were phrased to incorporate the boundary of the past 12 months (e.g., since last May 1) to aid patient recall. Patients were subsequently asked to report the approximate month and year of the visit and the reason and nature of the visits. Due to the large number of ED and hospital facilities within the Chicago area, self-report was considered the most accurate method to obtain these outcomes, as patient's medical records would not capture all urgent visits. Any reported ED visits that resulted in inpatient stays were counted only as an inpatient hospitalization, and only unplanned hospitalizations were included.

Covariates. A number of covariates that have well-established associations with the outcomes of interest were also collected. Health literacy was assessed using the Test of Functional Health Literacy in Adults (TOFHLA). ${ }^{16}$ The TOFHLA assesses comprehension of actual health information and is composed of a numeracy (17 items) and a literacy section (50 items). Scores range from 0 to 100 , with higher scores indicating higher health literacy. Scores are classified as limited $(0-74)$ or adequate $(75-100)$ health literacy. The MiniMental State Exam (MMSE) is a global measure of cognitive ability and is commonly used in medical settings, ${ }^{17}$ scores range from 0 to 30 , with higher scores indicating greater cognitive ability. Morbidity was defined as the number of self-reported prescription medications and chronic conditions (diabetes, chronic obstructive pulmonary disease, coronary vascular disease, congestive heart failure, asthma, hypertension, hypercholesterolemia, stroke, arthritis, cancer, depression). The number of prescription medications and chronic conditions was categorized as $0-1,2-4,5$, or more, and $0-1,2$, 3 , or more, respectively. Demographic characteristics included self-reported gender, age, race (White, African American, other), household income $(<\$ 25,000, \$ 25,000-\$ 49,000$, $>\$ 50,000$ ), and highest degree of education received (less than high school, high school graduate, some college, college graduate). To further characterize participants who identified unmet support needs, we also collected self-reported marital status and a measure of social isolation. Social isolation was assessed using the abbreviated (6-item) Lubben Social Network Scale (LSNS) ${ }^{18}$ which evaluates the number of familial and friend ties among an older adult population; ${ }^{19}$ scores less than 12 are classified as at risk of social isolation. ${ }^{18}$

\section{Analysis Plan}

$T$ tests and chi-square statistics were conducted to examine differences in demographic characteristics and study outcomes (health status, urgent healthcare utilization) by unmet tangible support needs (adequate vs. unmet), as appropriate. Multivariable linear and logistic regression models were conducted to examine predictors of health status (physical function, depressive symptoms, anxiety symptoms) and urgent healthcare utilization (ED visits, hospitalization). All models included unmet need, health literacy, cognitive ability, social isolation, self-reported age, race, number of chronic conditions, gender, income, and educational attainment. We also repeated the bivariable and multivariable regression analyses to conduct exploratory analyses among those who identified needing assistance in managing their health; we compared those who received sufficient help with those who reported unmet support needs. All analyses were performed using STATA 13.0 (College Station, TX).

\section{RESULTS}

The mean age of participants was $68.6(\mathrm{SD}=5.3)$ years and the majority $(71 \%)$ were female. The sample varied in terms of race and socioeconomic position; approximately half reported household incomes of less than $\$ 50,000$ annually and the highest degree of educational attainment as a high school degree or less, and a third of sample identified as African American (Table 1). A quarter of the sample were classified as having low health literacy. Participants had an average of three chronic conditions and were prescribed 4 daily medications. Very few were classified as socially isolated (18\%), and less than half (41\%) were married.

The majority $(76 \%, n=358)$ of participants reported they did not need assistance managing their health, $8 \%(n=37)$ reported they received sufficient help, 9\% $(n=41)$ reported they needed more help, and 7\% $(n=33)$ reported they needed much more help. Overall, $16 \%$ identified unmet support needs. Individuals with unmet support needs were more likely to be female $(82.4 \%$ vs. $69.1 \%)$, lower income $(60.9 \%$ vs. $26.1 \%$ ), African American (55.4\% vs. $33.9 \%$ ), have limited health literacy ( $43.8 \%$ vs. $24.5 \%)$, diagnosed with 3 or more chronic conditions $(79.7 \%$ vs. $55.7 \%)$, take 5 or more prescription medications ( $56.8 \%$ vs. $28.9 \%$ ), be unmarried ( $73.0 \%$ vs. $56.5 \%)$, or socially isolated $(27.1 \%$ vs. $16.3 \%)$ compared with individuals with adequate support (Table 1).

Significant differences by unmet support needs were found for all outcomes in unadjusted analyses (Table 2). Participants with unmet support needs had a mean $(M)$ physical function score of $39.0(\mathrm{SD}=6.7)$, which is equivalent to one standard deviation below the general US population norm, and was significantly lower than those with met support needs. Individuals with unmet support needs also reported a greater number of depressive $(M=$ $49.9(\mathrm{SD}=8.7)$ vs. $M=45.4(\mathrm{SD}=7.9), p<0.001)$ and anxiety symptoms $(M=55.8(\mathrm{SD}=8.2)$ vs. $M=50.3(\mathrm{SD}=8.7)$, $p<0.001)$ compared with individuals with adequate support. Individuals with unmet support needs were more likely to report an emergency department visit ( $46.0 \%$ vs. $14.4 \%, p<0.001)$ and hospitalization $(39.2 \%$ vs. $12.2 \%, p<0.001)$ in the past 12 months compared with individuals with adequate support.

Similar results were found in adjusted analyses (Table 3). Compared with individuals with adequate support, those with unmet support needs reported worse physical function $(\beta-$ $6.32 ; 95 \%$ CI $-8.31,-4.34, p<0.001)$, more anxiety 
Table 1 Participant Demographics by Adequacy of Social Support

\begin{tabular}{|c|c|c|c|c|}
\hline \multirow[t]{2}{*}{ Variable } & \multirow{2}{*}{$\begin{array}{l}\text { All } \\
\text { participants } \\
(N=469)\end{array}$} & \multicolumn{2}{|c|}{ Social support } & \multirow{2}{*}{$\begin{array}{l}P \\
\text { value }\end{array}$} \\
\hline & & $\begin{array}{l}\text { Adequate } \\
(n=395)\end{array}$ & $\begin{array}{l}\text { Unmet } \\
\text { needs } \\
(n=74)\end{array}$ & \\
\hline Female (\%) & 71.2 & 69.1 & 82.4 & 0.02 \\
\hline \multicolumn{5}{|l|}{ Age $(\%)$} \\
\hline $60-64$ & 28.2 & 27.3 & 32.4 & \multirow[t]{3}{*}{0.56} \\
\hline $65-74$ & 55.0 & 55.2 & 54.1 & \\
\hline $75+$ & 16.8 & 17.5 & 13.5 & \\
\hline \multicolumn{4}{|l|}{$\begin{array}{l}\text { Educational } \\
\text { attainment }(\%)\end{array}$} & \multirow[t]{5}{*}{$<0.001$} \\
\hline $\begin{array}{l}\text { Less than high } \\
\text { school }\end{array}$ & 9.8 & 9.1 & 13.5 & \\
\hline $\begin{array}{l}\text { High school } \\
\text { graduate }\end{array}$ & 12.2 & 13.4 & 5.4 & \\
\hline Some college & 21.8 & 17.7 & 43.2 & \\
\hline College graduate & 56.3 & 59.8 & 37.8 & \\
\hline \multicolumn{4}{|l|}{ Income $(\%)$} & \multirow[t]{4}{*}{$<0.001$} \\
\hline$<\$ 25,000$ & 31.4 & 26.1 & 60.9 & \\
\hline$\$ 25,000-49,999$ & 19.9 & 20.9 & 14.5 & \\
\hline \multirow{2}{*}{\multicolumn{4}{|c|}{ Race $(\%)$}} & \\
\hline & & & & \multirow[t]{2}{*}{0.001} \\
\hline African & 37.4 & 33.9 & 55.4 & \\
\hline \multicolumn{5}{|l|}{ American } \\
\hline White & 52.8 & 56.7 & 32.4 & \\
\hline Other & 9.8 & 9.4 & 12.2 & \\
\hline $\begin{array}{l}\text { MMSE score, } \\
\text { mean (SD) }\end{array}$ & $27.3(5.1)$ & $27.6(4.7)$ & $\begin{array}{l}26.1 \\
(6.7)\end{array}$ & 0.02 \\
\hline \multicolumn{4}{|l|}{ Health literacy $(\%)$} & \multirow[t]{3}{*}{0.001} \\
\hline Adequate & 72.5 & 75.5 & 56.2 & \\
\hline Limited & 27.5 & 24.5 & 43.8 & \\
\hline \multicolumn{4}{|l|}{$\begin{array}{l}\text { No. of chronic } \\
\text { conditions }(\%)\end{array}$} & \multirow[t]{4}{*}{0.001} \\
\hline $0-1$ & 19.4 & 21.5 & 8.1 & \\
\hline 2 & 21.1 & 22.8 & 12.2 & \\
\hline $3+$ & 59.5 & 55.7 & 79.7 & \\
\hline \multicolumn{4}{|l|}{$\begin{array}{l}\text { No. of prescription } \\
\text { medicines }(\%)\end{array}$} & \multirow[t]{6}{*}{$<0.001$} \\
\hline $0-1$ & 25.6 & 27.9 & 13.5 & \\
\hline $2-4$ & 41.2 & 43.3 & 29.7 & \\
\hline $5+$ & 33.3 & 28.9 & 56.8 & \\
\hline Married (\%) & 40.9 & 43.5 & 27.0 & \\
\hline Socially isolated (\%) & 18.0 & 16.3 & 27.1 & \\
\hline
\end{tabular}

symptoms ( $\beta 3.84 ; 95 \%$ CI $1.51,6.17, p=0.001)$, and more depressive symptoms ( $\beta$ 2.46; 95\% CI 0.32, 4.59, $p=0.02$ ). Across the three multivariable models of physical and mental health, male gender and higher income remained significant predictors of better physical function scores and less anxiety or depressive symptoms. Older adults reported fewer anxiety or depressive symptoms compared with adults between the ages of 60 and 64 and those who identified as African American reported fewer depressive symptoms compared with those who identified as White, and individuals classified as socially isolated reported more depressive symptoms. Individuals diagnosed with three or more chronic conditions or taking five or more prescription medicines had worse physical function and greater depressive symptoms compared with those with $\leq$ 1 chronic condition or prescription medication, respectively.

Individuals with unmet support needs also reported greater urgent healthcare utilization (ED: OR 2.86; 95\% CI 1.52, 5.41, $p=0.001$; hospitalization: OR 3.76 ; $95 \%$ CI $1.88,7.50$, $p<0.001)$ compared with individuals with adequate support.
Table 2 Unadjusted Associations Between Adequacy of Social Support and Health Status and Urgent Healthcare Use

\begin{tabular}{|c|c|c|c|c|}
\hline \multirow[t]{2}{*}{ Variable } & \multirow{2}{*}{$\begin{array}{l}\text { All } \\
\text { participants } \\
(N=469)\end{array}$} & \multicolumn{2}{|c|}{ Social support } & \multirow{2}{*}{$\begin{array}{l}P \\
\text { value }\end{array}$} \\
\hline & & $\begin{array}{l}\text { Adequate } \\
(n=395)\end{array}$ & $\begin{array}{l}\text { Unmet } \\
\text { needs } \\
(n=74)\end{array}$ & \\
\hline \multicolumn{5}{|c|}{ Health status (mean, SD) } \\
\hline $\begin{array}{l}\text { Physical } \\
\text { function score }\end{array}$ & $47.6(9.1)$ & $49.2(8.5)$ & $\begin{array}{l}39.0 \\
(6.7)\end{array}$ & $<0.001$ \\
\hline $\begin{array}{l}\text { Anxiety } \\
\text { symptoms }\end{array}$ & $51.2(8.8)$ & $50.3(8.7)$ & $\begin{array}{l}55.8 \\
(8.2)\end{array}$ & $<0.001$ \\
\hline $\begin{array}{l}\text { Depressive } \\
\text { symptoms }\end{array}$ & $46.1(8.2)$ & $45.4(7.9)$ & $\begin{array}{l}49.9 \\
(8.7)\end{array}$ & $<0.001$ \\
\hline \multicolumn{5}{|c|}{ Urgent healthcare use $(\%)$} \\
\hline $\begin{array}{l}\text { Emergency } \\
\text { department }\end{array}$ & 19.4 & 14.4 & 46.0 & $<0.001$ \\
\hline Hospitalization & 16.4 & 12.2 & 39.2 & $<0.001$ \\
\hline
\end{tabular}

Within the fully adjusted models, unmet support needs were the sole significant predictor, and all other covariates were not predictive of urgent healthcare utilization.

We conducted exploratory analyses among those who identified needing assistance in managing their health $(n=111)$ to investigate associations between those who received sufficient help with those who reported unmet support needs. Compared with those who received sufficient help, those who reported unmet support needs had worse physical function $(M=49.9(\mathrm{SD}=8.7)$ vs. $M=44.5$ (SD =9.2), $p<0.001)$, a greater number of anxiety symptoms $(M=55.8(\mathrm{SD}=8.2)$ vs. $M=52.6$ $(\mathrm{SD}=7.4), p=.04)$, and were more likely to report an emergency department visit in the past 12 months ( $46.0 \%$ vs. $21.6 \%, p=0.01)$. No differences were observed between number of depressive symptoms $(M=$ $49.9(\mathrm{SD}=8.7)$ vs. $M=47.9(\mathrm{SD}=7.9), p=0.24)$ and hospitalization $(39.2 \%$ vs. $37.8 \%, p=0.89)$. In adjusted analyses, no significant differences were observed.

\section{CONCLUSIONS}

In our sample, we found that nearly 1 in 6 community-dwelling older adults reported unmet tangible social support needs, and that unmet support needs were associated with greater urgent

Table 3 Adjusted Associations Between Adequacy of Social Support and Health Status and Urgent Healthcare Use

\begin{tabular}{ll}
\hline \hline Variable & Unmet social support \\
\hline Health status & $\beta(95 \% \mathrm{CI})$ \\
Physical function score & $-6.32(-8.31,-4.35)^{\ddagger}$ \\
Anxiety symptoms & $3.84(1.51,6.17)^{\dagger}$ \\
Depressive symptoms & $2.46(0.32,4.59)^{*}$ \\
Urgent healthcare use & OR $(95 \% \mathrm{CI})$ \\
Emergency department & $2.86(1.52,5.41)^{\dagger}$ \\
Hospitalization & $3.76(1.88,7.50)^{\ddagger}$ \\
\hline$* p<0.05$ & \\
$t p<0.01$ & \\
$t p<0.001$ &
\end{tabular}


healthcare use and worse health status. We found that individuals with unmet support needs, even after adjusting for many well-recognized covariates including social isolation, scored 6 points lower on the physical functioning score, and approximately 3.5 points greater with regard to anxiety symptoms, each suggesting a clinically meaningful difference from the overall sample. ${ }^{15}$ These differences are sizeable enough to inform the clinical care of patients and further underscores the importance of perceived adequacy of support on health.

These findings are an important contribution to the extensive body of literature on social relationships. While many facets of social relationships have been specified, the field has not readily incorporated aspects related to adequacy of support. ${ }^{6}$ Notably, perceived adequacy of tangible support was distinct from social isolation, which was only associated with depressive symptoms in the fully adjusted models. The literature on social support has operated under the assumption that if available, the support that is provided is sufficient to meet an individual's needs. ${ }^{20}$ This aspect is important as the quality of social relationships experienced by individuals is influenced by broader social and structural forces. ${ }^{21-23}$ These findings may reflect the principle of network homophily, which suggests that individuals disproportionately form network ties with others of similar background, thus the network members of disadvantaged individuals may also be in poor health. ${ }^{23,24}$ Therefore, while an individual may have an extensive social network, that network may be unable to provide the sufficient level of assistance an individual may require to engage in health-promoting behaviors, resulting in unmet tangible social support needs.

Remarkably, two straight forward questions were associated with health status and urgent healthcare use. While these two items do not provide explicit guidance to the nature of the need, this brief screener could be easily implemented and clinically useful for healthcare providers to identify individuals who may be at risk for poor outcomes, especially as there is growing recognition about the importance of screening for social determinants of health. ${ }^{25}$ Similar to the two-item Patient Health Questionnaire (PHQ-2), which has been incorporated into routine clinic intake procedures and is able to easily identify patients at risk for depression and notify clinic staff for additional follow-up, these two items could also be assessed as similar vital signs. Yet in order to be effective, health systems must have systems in place to respond after identifying individuals with unmet needs. ${ }^{26}$ As ambulatory care practices incorporate more allied health professionals including social workers and care coordinators, these professionals may be able to further work with patients to identify areas of need and provide a patient-tailored response or make appropriate referrals. Partnerships between clinics and community-based or public health organizations may also facilitate addressing these unmet social support needs.

Overall, unadjusted exploratory analyses observed a statistically significant difference in physical function scores, anxiety symptoms, and emergency department visits between those who received sufficient help with those who identified unmet support needs. These analyses were exploratory, due to the small sample sizes in each category (received sufficient help, $n=37$; unmet support needs, $n=74$ ) and future research should further explore differences between these two categories.

Older adults receive help from both formal and informal sources of support. Literature regarding informal caregiving of chronically ill adults has found that those who assume these roles often receive little to no training. ${ }^{27,}{ }^{28}$ Successful interventions related to developing caregivers skills seek to improve a sense of control, mastery and self-efficacy by reducing the caregiver's uncertainty. ${ }^{27,}{ }^{29}$ Complicating the development of these skills is the variability and sometimes unpredictable nature of these conditions, and therefore interventions are most successful when they are tailored to the key needs of specific patients and caregivers. ${ }^{29}$ Future research should evaluate whether caregiver training interventions are able to decrease older adults unmet tangible support needs. Additionally, home health aides, certified nursing assistants, and personal care aides provide the majority of formal care to older adults; however, these positions experience significant attrition and staffing insufficiencies due to low wages, inadequate training and supervision, and the perception of the position as a "dead-end" job, ${ }^{30}$ and as a result these service providers may be inadequately prepared to provide the requisite level of support these older adults need. A greater investment of resources is needed to support direct care workers as they provide paid care for older adults.

Our study should be recognized in the context of several limitations. Our findings are limited to English-speaking, predominantly female, older adults who are connected to primary care practices in one urban city. Additionally, our sample was predominantly comprised of individuals who self-identified as White or African American, and therefore did not include a large proportion of individuals who identified as other racial or ethnic backgrounds. The cross-sectional nature of these analyses limits inferences regarding directionality and causality; it is therefore possible that individuals with greater urgent healthcare utilization or worse health status may have a greater need for support, and subsequently unmet social support needs. Additionally, our measures of urgent healthcare utilization were self-reported; however, a high degree of concordance has previously been found among Medicare claims and older adults self-report of hospitalization. ${ }^{31}$ Our primary independent variable was assessed with only two items and did not provide additional detail regarding the insufficiency of the desired support. However, for the purpose of clinical utility, its brevity is a strength. Furthermore, we assessed perceived support, as opposed to received support; however, perceived support has previously been found to be more predictive of health outcomes. ${ }^{5,32}$ Lastly, there was a temporal mismatch between the assessment of unmet support needs (previous 6 months) and urgent healthcare utilization (previous 12 months), and therefore we may not have captured a need for support that occurred 6-12 months prior which may have been associated with an urgent healthcare visit. 
Future research should examine the adequacy of tangible support over time. As older adults are diagnosed with new illnesses or experience changes in health, their dependence on their support network may grow and the support person's ability to maintain a high level of assistance may not be able to keep up with the changes. ${ }^{29}$ Based on longitudinal findings, clinical encounters may want to regularly check the availability and adequacy of support.

Current medical and public health efforts aim to promote aging in place for older adults. Following acute care visits or declines in functional health, in order for older adults to continue to manage their health independently, it is often assumed that a support network will help older adults manage their health, but systems to develop these skills among older adults' support network are largely missing. Clinical and community organizations should partner to identify and address unmet tangible social support needs and work with older adults' support networks to understand their strengths and barriers to providing care for older adults. These findings highlight the importance of considering the adequacy of the support provided to older adults as they seek to manage their health independently.

Financial Support: This work was supported by a research grant from the National Institute on Aging (R01AG030611).

Corresponding Author: Rachel O'Conor, $\mathrm{PhD}, \mathrm{MPH}$; Division of General Internal Medicine and Geriatrics, Feinberg School of Medicine, Northwestern University, Chicago, IL, USA (e-mail: $r$ oconor@northwestern.edu).

\section{Compliance with Ethical Standards:}

The study was approved by the Institutional Review Board at Northwestern University Feinberg School of Medicine.

Conflict of Interest: Dr. Michael Wolf reports receiving consultancy fees from Abbot, Abbvie, Luto, Merck, United HealthCare, and Vivus, as well as research grants from Abbot, Abbvie, Merck, and United HealthCare. All remaining authors declare that they do not have a conflict of interest.

Publisher's Note: Springer Nature remains neutral with regard to jurisdictional claims in published maps and institutional affiliations.

\section{REFERENCES}

1. Gerteis J, Izrael D, Deitz D, LeRoy L, Ricciardi R, Miller T, et al. Multiple Chronic Conditions Chartbook. AHRQ Publications No. Q140038. Rockville, MD: Agency for healthcare Research and Quality; 2014.

2. Ward BW. Prevalence of Multiple Chronic Conditions among US Adults: Estimates from the National Health Interview Survey, 2010. Prev Chronic Dis 2013;10.

3. Gallant MP. The Influence of Social Support on Chronic Illness SelfManagement: A Review and Directions for Research. Health Educ Behav 2003;30(2): 170-95.

4. DiMatteo MR. Social support and patient adherence to medical treatment: a meta-analysis. Health Psychol 2004;23(2):207.

5. Holt-Lunstad J, Smith TB, Layton JB. Social relationships and mortality risk: a meta-analytic review. PLoS Med 2010;7(7):e1000316.

6. Holt-Lunstad J, Uchino BN. Social Support and Health. In: Glanz K, Rimer BK, Viswanath K, eds. Health Behavior: Theory, Research, and Practice. John Wiley \& Sons; 2015: 183-242.

7. Valtorta NK, Moore DC, Barron L, Stow D, Hanratty B. Older Adults' Social Relationships and Health Care Utilization: A Systematic Review. Am J Public Health 2018;108(4):e1-e10.
8. National Academies of Sciences, Engineering, MedicineAccounting for Social Risk Factors in Medicare Payment: Identifying Social Risk Factors. Washington, DC: National Academies Press; 2017.

9. Woloshin S, Schwartz LM, Tosteson AN, Chang CH, Wright B, Plohman J, et al. Perceived adequacy of tangible social support and health outcomes in patients with coronary artery disease. J Gen Intern Med 1997;12(10):613-8.

10. Song Y, Song H-J, Han H-R, Park S-Y, Nam S, Kim MT. Unmet Needs for Social Support and Effects on Diabetes Self-care Activities in Korean Americans With Type 2 Diabetes. Diabetes Educ 2012;38(1):77-85.

11. Wolf MS, Curtis LM, Wilson EA, Revelle W, Waite KR, Smith SG, et al. Literacy, cognitive function, and health: results of the LitCog study. J Gen Intern Med 2012;27(10):1300-7.

12. O'Conor R, Smith SG, Curtis LM, Benavente JY, Vicencio DP, Wolf MS. Mild Visual Impairment and Its Impact on Self-Care Among Older Adults. J Aging Health 2016

13. Callahan CM, Unverzagt FW, Hui SL, Perkins AJ, Hendrie HC. Six-item screener to identify cognitive impairment among potential subjects for clinical research. Med Care 2002;40(9):771-81.

14. Cella D, Riley W, Stone A, Rothrock N, Reeve B, Yount S, et al. The Patient-Reported Outcomes Measurement Information System (PROMIS) developed and tested its first wave of adult self-reported health outcome item banks: 2005-2008. J Clin Epidemiol 2010;63(11):1179-94.

15. Yost KJ, Eton DT, Garcia SF, Cella D. Minimally important differences were estimated for six Patient-Reported Outcomes Measurement Information System-Cancer scales in advanced-stage cancer patients. J Clin Epidemiol 2011;64(5):507-16.

16. Parker RM, Baker DW, Williams MV, Nurss JR. The test of functional health literacy in adults: a new instrument for measuring patients' literacy skills. J Gen Intern Med 1995;10(10):537-41.

17. Folstein MF, Folstein SE, McHugh PR. "Mini-mental state": a practical method for grading the cognitive state of patients for the clinician. J Psychiatr Res 1975;12(3):189-98.

18. Lubben J, Blozik E, Gillmann G, Iliffe S, von Renteln Kruse W, Beck JC, et al. Performance of an abbreviated version of the Lubben Social Network Scale among three European community-dwelling older adult populations. Gerontologist. 2006;46(4):503-13.

19. Lubben JE. Assessing social networks among elderly populations. Fam Community Health 1988;11(3):42-52.

20. Wolff JL, Feder J, Schulz R. Supporting Family Caregivers of Older Americans. N Engl J Med 2016;375(26):2513-5.

21. House JS, Landis KR, Umberson D. Social relationships and health. Science. 1988;241(4865):540.

22. Solar O, Irwin A. A Conceptual Framework for Action on the Social Determinants of Health. Geneva: World Health Organization. 2010.

23. McPherson M, Smith-Lovin L, Cook JM. Birds of a feather: Homophily in social networks. Annu Rev Sociol 2001;27(1):415-44.

24. Cornwell B. Social Disadvantage and Network Turnover. J Gerontol B Psychol Sci Soc Sci 2015;70(1): 132-42.

25. Diez Roux AV, Katz M, Crews DC, Ross D, Adler N. Social and Behavioral Information in Electronic Health Records: New Opportunities for Medicine and Public HealthAm J Prev Med 2015;49(6):980-3.

26. Garg A, Boynton-Jarrett R, Dworkin PH. Avoiding the unintended consequences of screening for social determinants of health. JAMA. 2016;316(8):813-4.

27. Given B, Sherwood PR, Given CW. What knowledge and skills do caregivers need? J Soc Work Educ 2008;44(sup3):115-23.

28. Reinhard SC, Levine C, Samis S. Home alone: Family caregivers providing complex chronic care: AARP Public Policy Institute Washington, DC; 2012.

29. Revenson T, Griva K, Luszczynska A, Morrison V, Panagopoulou E, Vilchinsky $\mathbf{N}$, et al. Caregiving in the Illness Context: Palgrave Macmillian; London; 2016.

30. Rowe JW, Fulmer T, Fried L. Preparing for better health and health care for an aging population. JAMA. 2016;316(16):1643-4.

31. Wolinsky FD, Miller TR, An H, et al. Hospital episodes and physician visits: the concordance between self-reports and medicare claims. Med Care 2007;45(4):300-307.

32. Uchino BN. Understanding the links between social support and physical health: A life-span perspective with emphasis on the separability of perceived and received support. Perspect Psychol Sci 2009;4(3):236-55.

Publisher's Note Springer Nature remains neutral with regard to jurisdictional claims in published maps and institutional affiliations. 